\title{
Energy-Optimal Cooperative Manipulation via Provable Internal-Force Regulation
}

\author{
Christos K. Verginis and Dimos V. Dimarogonas
}

\begin{abstract}
This paper considers the optimal cooperative robotic manipulation problem in terms of energy resources. In particular, we consider rigid cooperative manipulation systems, i.e., with rigid grasping contacts, and study energy-optimal conditions in the sense of minimization of the arising internal forces, which are inter-agent forces that do not contribute to object motion. Firstly, we use recent results to derive a closed form expression for the internal forces. Secondly, by using a standard inverse dynamics control protocol, we provide novel conditions on the force distribution to the robotic agents for provable internal force minimization. Moreover, we derive novel results on the provable achievement of a desired non-zero interagent internal force vector. Extensive simulation results in a realistic environment verify the theoretical analysis.
\end{abstract}

\section{INTRODUCTION}

Multi-agent robotic systems have received a considerable amount of attention during the last decades, due to the advantages they offer with respect to single-agent setups. Especially in the case of robotic manipulation of payloads, multi-agent frameworks can yield significant advantages due to the potentially heavy payloads or challenging maneuvers.

Cooperative robotic manipulation has been extensively studied in the related literature. Most works consider decentralized schemes, where there is no communication between the agents, and use impedance and/or force control [1][4], possibly with force/torque measurements at the grasping points (e.g., [5], [6]). In addition, numerous works consider unknown dynamics/kinematics of the agents and the object and/or external disturbances, which they compensate for via adaptive and robust control techniques [7]-[10].

An important property in rigid cooperative manipulation systems (i.e., when the grasps are rigid) that has been studied thoroughly in the related literature is the regulation of internal forces. Internal forces are forces exerted by the agents at the grasping points that do not contribute to the motion of the object. While a certain amount of such forces is required in many cases (e.g., to avoid contact loss in multi-fingered manipulation), they need to be minimized in order to prevent object damage and unnecessary effort of the agents. In fact, minimization of internal forces yields optimal cooperative manipulation schemes in terms of energy resources, promoting thus environmental sustainability,

C. K. Verginis and D. V. Dimarogonas are with the KTH Center of Autonomous Systems, School of Electrical Engineering and Computer Science, KTH Royal Institute of Technology, SE-100 44, Stockholm, Sweden. Email: \{cverginis, dimos\}akth. se.This work was supported by the H2020 ERC Starting Grant BUCOPHSYS, the European Union's Horizon 2020 Research and Innovation Programme under the GA No. 731869 (Co4Robots), the Swedish Research Council (VR), the Knut och Alice Wallenberg Foundation (KAW) and the Swedish Foundation for Strategic Research (SSF). which is becoming more and more indispensable over the years (robotic manipulation studies have focused on energybased criteria, e.g., [11]).

More specifically, the most efficient way of manipulating an object, in terms of energy resources, is for the robotic agents to not exert internal forces, i.e., forces that cancel each other out, and exert only forces that move the object. Consider the extreme example of a leader agent working towards bringing an object to a desired location, whereas the other agents have zero inputs. Since the grasps are rigid, a sufficiently powerful force by the leader will achieve the task by "dragging" the other agents along, compensating for their dynamics, and creating significant internal forces. Hence, from a control perspective, the goal of rigid cooperative manipulation schemes should be the design of a control protocol that achieves a desired cooperative manipulation task, while guaranteeing that the internal forces are zero.

Most works in rigid cooperative manipulation assume a certain decomposition of the exerted forces in motioninducing and internal ones, without explicitly showing that the actual internal forces will be indeed regulated to the desired ones (e.g., [2], [5], [6]); [12]-[15] analyze specific load decompositions based on whether they provide internal force-free expressions, whereas [16] is concerned with the cooperative manipulation interaction dynamics. The decompositions in the aforementioned works, however, are based on the inter-agent distances and do not take into account the actual dynamics of the agents. The latter, as we show, are tightly connected to the arising internal forces, which gives new insight on internal force-free cooperative manipulation.

More specifically, this paper considers the energy-optimal cooperative manipulation problem, in the sense of minimizing the arising inter-agent internal forces. We use recent results on the application of Gauss' principle on constraint systems to cooperative manipulation schemes in order to obtain a closed-form expression for the inter-agent internal forces. We combine then this expression with a standard inversedynamics control protocol to provide novel conditions on the agent force distribution for provable internal force-free cooperative manipulation, which achieves optimality in terms of energy resources. Moreover, we provide formal guarantees for the achievement of a potential desired value for the arising internal forces. Finally, extensive simulations results illustrate the theoretical findings.

\section{PROBLEM FORMULATION}

Consider $N$ robotic agents, indexed by the set $\mathcal{N}:=$ $\{1, \ldots, N\}$, rigidly grasping a rigid object, as shown in Fig. 


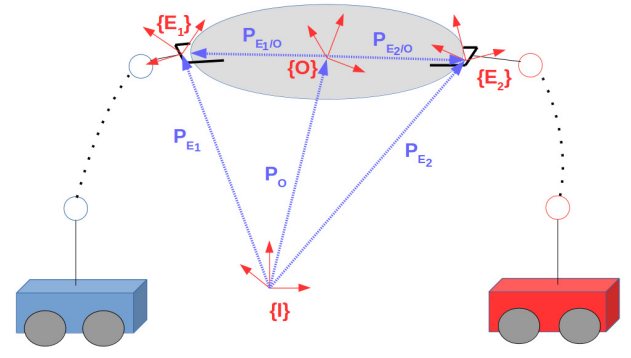

Fig. 1. Two robotic agents rigidly grasping an object.

1. We denote by $q_{i}, \dot{q}_{i} \in \mathbb{R}^{n_{i}}$, with $n_{i} \in \mathbb{N}, \forall i \in \mathcal{N}$, the $i$ th agent's generalized joint-space variables. We denote the inertial position and orientation of the $i$ th end-effector by $p_{i}:=p_{i}\left(q_{i}\right)$ and $\eta_{i}:=\eta_{i}\left(q_{i}\right)$, respectively, obtained by the forward kinematics, with $x_{i}:=x_{i}\left(q_{i}\right):=\left[p_{i}^{\top}, \eta_{i}^{\top}\right]^{\top} \in$ $\mathbb{M}:=\mathbb{R}^{3} \times \mathbb{T}$, where $\mathbb{T}$ is an appropriate orientation space. Similarly, the velocity of the $i$ th end-effector is denoted by $v_{i}:=\left[\dot{p}_{i}^{\top}, \omega_{i}^{\top}\right]^{\top}$, where $\omega_{i} \in \mathbb{R}^{3}$ is the respective angular velocity, and it holds that $v_{i}=J_{i}\left(q_{i}\right) \dot{q}_{i}$, where $J_{i}: \mathbb{S}_{i} \rightarrow$ $\mathbb{R}^{6 \times n_{i}}$ is the manipulator Jacobian, and $\mathbb{S}_{i}:=\left\{x_{i}\left(q_{i}\right) \in \mathbb{M}\right.$ : $\left.\operatorname{dim}\left(\operatorname{null}\left(J_{i}\left(q_{i}\right)\right)\right)=0\right\}$ is the set away from singularities in task-space, $\forall i \in \mathcal{N}$. Moreover $R_{i}\left(\eta_{i}\right) \in \mathrm{SO}(3)$ is the $i$ th end-effector's rotation matrix, $\forall i \in \mathcal{N}$, where $\mathrm{SO}(3)$ is the $3 \mathrm{D}$ special orthogonal group. The task-space dynamics of each agent is [17]:

$$
M_{i}\left(x_{i}\right) \dot{v}_{i}+C_{i}\left(x_{i}, \dot{x}_{i}\right) v_{i}+g_{i}\left(x_{i}\right)=u_{i}-h_{i},
$$

where $M_{i}: \mathbb{S}_{i} \rightarrow \mathbb{R}^{6 \times 6}$ is the positive definite inertia matrix, $C_{i}: \mathbb{S}_{i} \times \mathbb{R}^{6} \rightarrow \mathbb{R}^{6 \times 6}$ is the Coriolis matrix, $g_{i}: \mathbb{S}_{i} \rightarrow \mathbb{R}^{6}$ is the gravity vector, and $u_{i} \in \mathbb{R}^{6}$ is the task space wrench, related to the torque actuation $\tau_{i} \in \mathbb{R}^{n_{i}}$ of agent $i$ as $\tau_{i}=$ $J_{i}^{\top} u_{i}, i \in \mathcal{N}$. Moreover, $h_{i} \in \mathbb{R}^{6}$ are the forces exerted by the agents to the object at the grasping points, $\forall i \in \mathcal{N}$. The aforementioned dynamics can be stacked as:

$$
M(x) \dot{v}+C(x, \dot{x}) v+g(x)=u-h,
$$

with $M:=\operatorname{diag}\left\{\left[M_{i}\right]_{i \in \mathcal{N}}\right\}, C:=\operatorname{diag}\left\{\left[C_{i}\right]_{i \in \mathcal{N}}\right\} \in$ $\mathbb{R}^{6 N \times 6 N}, v:=\left[v_{1}^{\top}, \ldots, v_{N}^{\top}\right], x:=\left[x_{1}^{\top}, \ldots, x_{N}^{\top}\right]^{\top}, h:=$ $\left[h_{1}^{\top}, \ldots, h_{N}^{\top}\right]^{\top}, u:=\left[u_{1}^{\top}, \ldots, u_{N}^{\top}\right]^{\top}, g:=\left[g_{1}^{\top}, \ldots, g_{N}^{\top}\right]^{\top} \in$ $\mathbb{R}^{6 N}$.

Regarding the object, we denote by $x_{O}:=\left[p_{O}^{\top}, \eta_{O}^{\top}\right]^{\top} \in \mathbb{M}$, $v_{O}:=\left[\dot{p}_{O}^{\top}, \omega_{O}^{\top}\right]^{\top} \in \mathbb{R}^{6}$ the pose and generalized velocity of the object's center of mass; $R_{O}\left(\eta_{O}\right) \in \mathrm{SO}(3)$ is the object's rotation matrix. The object dynamics is described by

$$
\begin{aligned}
& \dot{R}_{O}=S\left(\omega_{O}\right) R_{O} \\
& M_{O}\left(x_{O}\right) \dot{v}_{O}+C_{O}\left(x_{O}, \dot{x}_{O}\right) v_{O}+g_{O}\left(x_{O}\right)=h_{O},
\end{aligned}
$$

where $M_{O}: \mathbb{M} \rightarrow \mathbb{R}^{6 \times 6}$ is the positive definite inertia matrix, $C_{O}: \mathbb{M} \times \mathbb{R}^{6} \rightarrow \mathbb{R}^{6 \times 6}$ is the Coriolis matrix, $g_{O}: \mathbb{M} \rightarrow \mathbb{R}^{6}$ is the gravity vector, $h_{O} \in \mathbb{R}^{6}$ is the vector of generalized forces acting on the object's center of mass, and $S(\cdot)$ is the skew-symmetric operator defined according to the cross product $S(a) b=a \times b$ for $a, b \in \mathbb{R}^{3}$.
In the rigid cooperative manipulation setting (see Fig. 1), the pose of the agents and the object's center of mass satisfy

$$
p_{i}=p_{O}+p_{i o}=p_{O}+R_{i} p_{i o}^{i},
$$

$\forall i \in \mathcal{N}$, where $p_{i O}:=p_{i}-p_{O}$, and $p_{i O}^{i}$, is the constant vector from the $i$ th grasping point to the object's center of mass. Differentiating (3), along with the fact that, due to the grasping rigidity, it holds that $\omega_{i}=\omega_{O}, \forall i \in \mathcal{N}$, one obtains

$$
v_{i}=J_{O_{i}}\left(x_{i}\right) v_{O},
$$

where $J_{O_{i}}: \mathbb{M} \rightarrow \mathbb{R}^{6 \times 6}$ is the object-to-agent Jacobian matrix, with

$$
J_{O_{i}}\left(x_{i}\right)=\left[\begin{array}{cc}
I_{3} & -S\left(p_{i O}\right) \\
0_{3 \times 3} & I_{3}
\end{array}\right], \forall x_{i} \in \mathbb{M},
$$

where $I_{n}$ is the $n \times n$ identity matrix, and $0_{n \times m}$ the $n \times m$ matrix of zeros. Note that $J_{O_{i}}$ is always full-rank, due to the rigidity of the grasping contacts. By stacking $J_{O_{i}}^{\top}$ we form the grasp matrix as

$$
G(x):=\left[J_{O_{1}}\left(x_{1}\right)^{\top}, \ldots, J_{O_{N}}\left(x_{N}\right)^{\top}\right] \in \mathbb{R}^{6 \times 6 N} .
$$

We can now write (4) in stack vector form as

$$
v=G(x)^{\top} v_{O} .
$$

The kineto-statics duality along with the grasp rigidity suggest that the object and agents forces $h_{O}, h$, are related as:

$$
h_{O}=G(x) h .
$$

By substituting (1) into (8) and then (2), we obtain the coupled dynamics:

$$
\widetilde{M}(\bar{x}) \dot{v}_{O}+\widetilde{C}(\bar{x}) v_{O}+\widetilde{g}(\bar{x})=G(q) u,
$$

where $\widetilde{M}:=M_{O}+G M G^{\top}, \widetilde{C}:=C_{O}+G C G^{\top}+G M \dot{G}^{\top}$, $\widetilde{g}:=g_{O}+G g, \bar{x}$ is the overall state $\bar{x}:=\left[x^{\top}, \dot{x}^{\top}, x_{O}^{\top}, \dot{x}_{O}^{\top}\right]^{\top} \in$ $\mathbb{M}^{2} \times \mathbb{R}^{6 N+6}$, and we have omitted the arguments for brevity.

The force vector $h$ that the agents exert at the grasping points can be decoupled into motion-induced $h_{\mathrm{m}}$ and internal forces $h_{\text {int }}$ as $h=h_{\mathrm{m}}+h_{\text {int }}$. The internal forces $h_{\text {int }}$ are forces that the agents exert to the object and belong to the nullspace of $G(x)$ (i.e., $G(x) h_{\text {int }}=0$ ). Hence, they do not contribute to the acceleration of the coupled system and result in internal stresses that might damage the object. A closed form analytic expression for $h_{\text {int }}$ will be given in Section III

As discussed in Section I the optimal - in terms of agent effort - way of cooperatively manipulating a rigidly grasped object is by regulating the internal forces to zero. In that way, each agent does not have to compensate for potential deviations of the other agents from the appropriate trajectory, which would require more unnecessary effort. On the other hand, in a more realistic setting, it might be desired for the agents to exert a small internal force, e.g., to maintain contact with the object. Therefore, the problem in hand is the design of control protocols, and more specifically force distributions to the agents, that provably regulate the internal forces to a desired value. By provably we mean that the results, in contrast to the works in the related literature, are based 
on derived closed-form expressions for the internal forces. In particular, by considering a standard inverse-dynamics control scheme, our main results, given in the next section, consist of the following: firstly, we provide novel necessary and sufficient conditions for the distribution of a desired object force to the agents, characterized by a pseudo-inverse $G^{*}$ of $G$, in order to achieve object motion free of internal forces. Secondly, we provide an appropriate modification of the control scheme that guarantees achievement of a desired nonzero internal force $h_{\text {int,d. }}$.

\section{MAIN RESULTS}

We first use previous works on Gauss's principle [18] to derive a closed-form expression for the internal forces $h_{\text {int }}$. Let the unconstrained dynamical system be $M(x) \alpha(x, \dot{x}):=$ $u-C(x, \dot{x}) v-g(x)$, where $\alpha(x, \dot{x})$ is the coupled system's unconstrained acceleration, i.e., the acceleration the agents would have if they were not grasping the object.

Next, from (4) and the fact that $J_{O_{i}}\left(x_{i}\right)$ is invertible, $\forall i \in$ $\mathcal{N}$, we obtain $v_{1}=J_{O_{1}}\left(x_{1}\right) J_{O_{i}}\left(x_{i}\right)^{-1} v_{j}=J_{i j}\left(x_{i}, x_{j}\right) v_{j}$, $\forall i \in\{2, \ldots, N\}$, where $J_{i j}: \mathbb{M}^{2} \rightarrow \mathbb{R}^{6 \times 6}$ is defined as

$$
J_{i j}\left(x_{i}, x_{j}\right):=\left[\begin{array}{cc}
I_{3} & -S\left(p_{i j}\right) \\
0 & I_{3}
\end{array}\right], \forall i, j \in \mathcal{N}, i \neq j,
$$

with $p_{i j}:=p_{i}-p_{j}$. The aforementioned expression can be written in compact form as $A(x, \dot{x}) v=0$, with

$$
\begin{aligned}
& A(x, \dot{x}):= \\
& {\left[\begin{array}{ccccccc}
I_{3} & S\left(p_{12}\right) & -I_{3} & 0_{3 \times 3} & \ldots & 0_{3 \times 3} & 0_{3 \times 3} \\
0_{3 \times 3} & I_{3} & 0_{3 \times 3} & -I_{3} & \ldots & 0_{3 \times 3} & 0_{3 \times 3} \\
\vdots & \vdots & \ldots & \ddots & \ddots & \vdots & \vdots \\
I_{3} & S\left(p_{1 N}\right) & 0_{3 \times 3} & 0_{3 \times 3} & \ldots & -I_{3} & 0_{3 \times 3} \\
0_{3 \times 3} & I_{3} & 0_{3 \times 3} & 0_{3 \times 3} & \ldots & 0_{3 \times 3} & -I_{3}
\end{array}\right] .}
\end{aligned}
$$

By differentiation, we obtain

$$
A(x, \dot{x}) \dot{v}=-\dot{A}(x, \dot{x}) v,
$$

which encodes the rigidity coupling constraints among the agents, induced by the rigidity contacts.

According to Gauss's principle [15], [18], the actual accelerations of the coupled system are the solutions of the constrained optimization problem

$$
\begin{array}{ll}
\min _{\dot{v}} & (\dot{v}-\alpha(x, \dot{x}))^{\top} M(x)(\dot{v}-\alpha(x, \dot{x})) \\
\text { s.t. } & A(x, \dot{x}) \dot{v}=-\dot{A}(x, \dot{x}) v .
\end{array}
$$

The resulting accelerations can be proven to be [15], [18]

$$
M(x) \dot{v}=u-C(x, \dot{x}) v-g(x)+h_{\text {int }},
$$

where $h_{\text {int }}$ are the resulting internal forces:

$$
h_{\text {int }}=-M^{\frac{1}{2}}\left(A M^{-\frac{1}{2}}\right)^{\dagger}(\dot{A} v+A \alpha),
$$

with ()$^{\dagger}$ denoting the Moore-Penrose pseudoinverse, and we have omitted the arguments for notational brevity. The aforementioned result has an intuitive interpretation. It states that the difference of the unconstrained and actual acceleration of the agents lies in the deviation $\dot{A} v+A \alpha$, associated with the constraints 10 , scaled by the term $M^{\frac{1}{2}}\left(A M^{-\frac{1}{2}}\right)^{\dagger}$. In other words, internal forces will arise if the unconstrained acceleration of the agents tends to violate the rigidity constraints. In fact, one concludes that no internal forces will occur if the term $\dot{A} v+A \alpha$ lies in the nullspace of the matrix $M^{\frac{1}{2}}\left(A M^{-\frac{1}{2}}\right)^{\dagger}$, which, since $M$ is positive definite, is the nullspace of $A^{\top}$ :

Corollary 1: The cooperative manipulation system is free of internal forces, i.e., $h_{\text {int }}=0$, if and only if

$\dot{A} v+A M^{-1}(u-C v-g) \in \operatorname{null}\left(M^{\frac{1}{2}}\left(A M^{-\frac{1}{2}}\right)^{\dagger}\right)=\operatorname{null}\left(A^{\top}\right)$

Next, we derive a novel result relating $A$ and $G$ that is important for the subsequent analysis:

Theorem 1: Let a rigid cooperative manipulation system with grasp and constraint matrix $G(x)$, and $A(x, \dot{x})$ as defined in (5) and (10), respectively. Then it holds that

$$
\operatorname{null}(G)=\operatorname{range}\left(A^{\top}\right)
$$

Proof: The range of $G^{\top}$ is $\operatorname{range}\left(G^{\top}\right)=\{\chi=$ $\left[\chi_{1}^{\top}, \ldots, \chi_{N}^{\top}\right]^{\top}: \chi_{i}=J_{O_{i}}\left(x_{i}\right) \chi_{o}, \forall x_{i} \in \mathbb{M}, \chi_{o} \in \mathbb{R}^{6}, i \in$ $\mathcal{N}\}$. Hence, for all $\chi \in \operatorname{range}\left(G^{\top}\right)$ it holds that $\chi_{1}=$ $J_{O_{1}}\left(x_{1}\right) \chi_{O}=J_{O_{1}}\left(x_{1}\right) J_{O_{i}}\left(x_{i}\right)^{-1} \chi_{i}, \forall i \in\{2, \ldots, N\}$, which is the set of vectors that form the nullspace of $A$. By invoking the rank-nullity theorem (Th. 2.10 of [19]), we complete the proof.

Therefore, one concludes that the internal forces are all the vectors $z$ for which there exists a vector $y$ such that $z=$ $A^{\top} y$. This can be also verified by (11); one can prove that $\operatorname{range}\left(M^{\frac{1}{2}}\left(A M^{-\frac{1}{2}}\right)^{\dagger}\right)=\operatorname{range}\left(A^{\top}\right)$.

Let now a desired bounded position and orientation trajectory for the object be dictated by the signals $p_{\mathrm{d}}: \mathbb{R}_{\geq 0} \rightarrow \mathbb{R}^{3}$ and $R_{\mathrm{d}}: \mathbb{R}_{>0} \rightarrow \mathrm{SO}(3)$, with bounded derivatives, such that $\dot{R}_{\mathrm{d}}=S\left(\omega_{\mathrm{d}}\right) R_{\mathrm{d}}$, where $\omega_{\mathrm{d}} \in \mathbb{R}^{3}$ is the desired angular velocity. A straightforward position error metric is $e_{p}:=$ $p_{O}-p_{\mathrm{d}}$, whereas for the orientation we choose [20] $e_{O}=$ $\frac{1}{2} \operatorname{trace}\left(I_{3}-R_{\mathrm{d}}^{\top} R_{O}\right) \in[0,2]$, which, after differentiation, using (2a) and properties of skew-symmetric matrices [20], becomes

$$
\dot{e}_{O}=\frac{1}{2} e_{R}^{\top} R_{O}^{\top}\left(\omega_{O}-\omega_{\mathrm{d}}\right),
$$

where $e_{R}:=S^{-1}\left(R_{\mathrm{d}}^{\top} R_{O}-R_{O}^{\top} R_{\mathrm{d}}\right)$. Then one can show that $e_{R}=0$ when $\operatorname{trace}\left(R_{\mathrm{d}}^{\top} R_{O}\right)=3$ or when $\operatorname{trace}\left(R_{\mathrm{d}}^{\top} R_{O}\right)=$ -1 . The second case represents an undesired equilibrium, where the desired and the actual orientation differ by 180 degrees. This issue is caused by topological obstructions on $\mathrm{SO}(3)$ and it has been proven that no continuous controller can achieve global stabilization. Therefore, we guarantee in the following convergence of $e_{R}$ to 0 , given that $e_{R}(0)<2$. Define also the stack error signals $e_{v}:=v_{O}-v_{\mathrm{d}}$, where $v_{\mathrm{d}}:=\left[\dot{p}_{\mathrm{d}}^{\top}, \omega_{\mathrm{d}}^{\top}\right]^{\top}$, and $e_{x}:=\left[e_{p}^{\top}, \frac{1}{2\left(2-e_{O}\right)^{2}} e_{R}^{\top} R_{O}^{\top}\right]$.

Let now $G^{*} \in \mathbb{R}^{6 N \times 6}$ be a right pseudo-inverse of $G$, i.e. $G G^{\star}=I_{6}$, which is used to distribute a desired object force to the agents (see (8)). Standard choices in the related literature are $G^{*}=G^{\top}\left(G G^{\top}\right)^{-1}$ or the weighted version $G^{*}=W G^{\top}\left(G W G^{\top}\right)^{-1}$ for a gain matrix $W \in \mathbb{R}^{6 N \times 6 N}$. 
A standard inverse dynamics control law [17] is

$$
\begin{aligned}
u= & u_{A}+g+\left(C G^{\top}+M \dot{G}^{\top}\right) v_{O}+G^{*}\left(g_{O}+C_{O} v_{O}\right)+ \\
& \left(M G^{\top}+G^{*} M_{O}\right) f_{O, \mathrm{~d}},
\end{aligned}
$$

where $f_{O, \mathrm{~d}}:=\dot{v}_{\mathrm{d}}-K_{v} e_{v}-K_{x} e_{x}, K_{x}:=\operatorname{diag}\left\{K_{p}, k_{R} I_{3}\right\}$, $K_{v} \in \mathbb{R}^{6 \times 6}, K_{p} \in \mathbb{R}^{3 \times 3}$ are positive definite and constant gain matrices, $k_{R}>0$ is a positive gain constant, and $u_{A}$ is a vector that belongs to the set $\left\{y \in \mathbb{R}^{6 N}: y \in\right.$ $\left.\operatorname{null}(G)\} \cap\left\{y \in \mathbb{R}^{6 N}: A M^{-1} y \in \operatorname{null}\left(A^{\top}\right)\right\}\right\}^{1}$ and is responsible for secondary tasks (e.g., singularity avoidance). The fact that $G u_{A}=0$ implies that $u_{A}$ does not contribute to object motion and the fact that $A M^{-1} u_{A} \in \operatorname{null}\left(A^{\top}\right)$ implies that it does not create internal forces. Note that $G^{*}$ distributes to the agents an implicit desired force to be applied to the object, i.e., $g_{O}+C_{O} v_{O}+M_{O} f_{O, \mathrm{~d}}$. It turns out, however, that in order for the system to be free of internal forces, $G^{*}$ cannot be chosen arbitrarily. In fact, $G^{*}$ must be directly linked with the constraint and inertia matrices, $A$ and $M$, respectively, as the next main theorem shows.

Theorem 2: Let $N$ robotic agents rigidly grasping an object, with coupled dynamics 99). Consider the control law (15) and assume that $e_{O}(0)<2$. Then the solution of the closed-loop system satisfies the following:

1) $e_{O}(t)<2, \forall t \geq 0$

2) $p_{O}(t)-p_{\mathrm{d}}(t) \rightarrow 0, R_{\mathrm{d}}(t)^{\top} R_{O}(t) \rightarrow I_{3}$,

3) It holds that $h_{\text {int }}=0$, if and only if

$$
\operatorname{range}\left(A M^{-1} G^{*}\right) \subseteq \operatorname{null}\left(A^{\top}\right) .
$$

Proof: By substituting the inverse-dynamics law (15) in (9) and using the facts that $G G^{*}=I_{6}, G u_{A}=0_{6}$, we obtain $\widetilde{M}(\bar{x})\left(\dot{e}_{v}+K_{v} e_{v}+K_{x} e_{x}\right)=0$, which, since $\widetilde{M}$ is positive definite, implies

$$
\dot{e}_{v}=-K_{v} e_{v}-K_{x} e_{x} .
$$

1) Consider now the Lyapunov-like function

$$
V:=\frac{1}{2} e_{p}^{\top} K_{p} e_{p}+\frac{k_{R}}{2-e_{O}}+\frac{1}{2} e_{v}^{\top} e_{v},
$$

for which it holds that $V(0)<\infty$, since $e_{O}(0)<2$. By differentiating $V$, and using (14) and (17), we obtain $\dot{V}=-e_{v}^{\top} K_{v} e_{v} \leq 0$. Hence, it holds that $V(t) \leq$ $V(0)<\infty$, implying that $\frac{k_{R}}{2-e_{O}(t)}$ is bounded and thus $e_{O}(t)<2, \forall t \geq 0$.

2) Since $V(t) \leq V(0)<\infty$, the errors $e_{p}$, $e_{v}$ are bounded, which, given the boundedness of the $p_{\mathrm{d}}$, $R_{\mathrm{d}}, \dot{p}_{\mathrm{d}}, \omega_{\mathrm{d}}$, implies the boundedness of $u$. Hence, it can be proven that $\ddot{V}$ is bounded, implying the uniform continuity of $\dot{V}$. Therefore, according to Barbalat's lemma ([21], Lemma 8.2), we deduce that $\lim _{t \rightarrow \infty} \dot{V}(t)=0 \Rightarrow \lim _{t \rightarrow \infty} e_{v}(t)=0$. Since $e_{x}(t)$ is also bounded, it can be proven by using the same arguments that $\lim _{t \rightarrow \infty} \dot{e}_{v}(t)=0$ and hence (17) implies that $\lim _{t \rightarrow \infty} e_{x}(t)=0$.

${ }^{1}$ An example for $u_{A}$ is given later, showing that this set is non-empty
3) The acceleration of the unconstrained system of the agents is, after substituting (15):

$$
\begin{gathered}
M \alpha=u_{A}+g+\left(C G^{\top}+M \dot{G}\right) v_{O}+M G^{\top} \alpha_{\mathrm{d}}+ \\
G^{*}\left(g_{O}+C_{O} v_{O}+M_{O} \alpha_{\mathrm{d}}\right)-C v-g,
\end{gathered}
$$

where $\alpha_{\mathrm{d}}:=\dot{v}_{\mathrm{d}}-K_{v} e_{v}-K_{x} e_{x}$, and hence, after substituting $v=G^{\top} v_{O}$, we obtain the internal forces, according to 11]:

$$
\begin{aligned}
h_{\text {int }}= & M^{\frac{1}{2}}\left(A M^{-\frac{1}{2}}\right)^{\dagger}\left[\dot{A} G^{\top} v_{O}+A \dot{G}^{\top} v_{O}+A G^{\top} \alpha_{\mathrm{d}}+\right. \\
& \left.A M^{-1} u_{A}+A M^{-1} G^{*}\left(g_{O}+C_{O} v_{O}+M_{O} \alpha_{\mathrm{d}}\right)\right] .
\end{aligned}
$$

According to Theorem 1 , it holds that $A G^{\top}=0$, which implies that $\dot{A} G^{\top}+A G^{\top}=0$. Therefore, by also using the fact that $A M^{-1} u_{A} \in \operatorname{null}\left(A^{\top}\right), h_{\text {int }}$ becomes

$$
h_{\text {int }}=M^{\frac{1}{2}}\left(A M^{-\frac{1}{2}}\right)^{\dagger}\left(A M^{-1} G^{*}\left(g_{O}+C_{O} v_{O}+M_{O} \alpha_{\mathrm{d}}\right)\right) \text {, }
$$

which is zero for all the vectors $g_{O}+C_{O} v_{O}+M_{O} \alpha_{\mathrm{d}}$ if and only if range $\left(A M^{-1} G^{*}\right) \subseteq \operatorname{null}\left(M^{\frac{1}{2}}\left(A M^{-\frac{1}{2}}\right)^{\dagger}\right)$, which is the same as null $\left(A^{\top}\right)$ (see Corollary 1 ).

Remark 1: Theorem 2 provably shows that 16 is a necessary and sufficient condition for a standard inverse dynamics control scheme to achieve zero internal forces and therefore energy-optimal cooperative object manipulation. According to the authors' best knowledge, this is the first time that such conditions are derived from the actual internal force expression (11). In fact, (16) gives guidelines for designing a force distribution $G^{*}$ without internal forces, and apparently this is achieved by incorporating the inertia matrix $M$. As discussed before, this is crucial for achieving energy-optimal cooperative manipulation, where the agents do not have to "waste" control input and hence energy resources that do not contribute to object motion. Related works that focus on deriving internal force-free distributions $G^{*}$, e.g., [12]-[15], are solely based on the inter-agent distances, neglecting the actual dynamics of the agents and the object. The expression (11), however, gives new insight on the topic and suggests that the dynamic terms of the system play a significant role in the arising internal forces, as also indicated by Corollary 1. This is further exploited by Theorem 2 to produce a new class of distributions $G^{*}$ for the control type of (15), which depends on the dynamics and more specifically on the inertia matrix of the system. In fact, it is proven in [22] that the unique choice that provably regulates internal forces to zero is $G^{*}=M G^{\top}\left(G M G^{\top}\right)^{-1}$.

Remark 2: Note that the employed inverse dynamics controller requires knowledge of the agent and object dynamics. In case of dynamic parameter uncertainty, standard adaptive control schemes that attempt to estimate potential uncertainties in the model (see, e.g., [8], [10]) would intrinsically create internal forces, since the dynamics of the system would not be accurately compensated. The same holds for schemes that employ force/torque sensors that provide the respective measurements at the grasp points (e.g., [5], [6]) in periodic time instants. Since the interaction forces depend explicitly on the control input, such measurements 
will unavoidably correspond to the interaction forces of the previous time instants due to causality reasons, creating thus small disturbances in the dynamic model. Nevertheless, it must be noted that in a realistic setting the dynamic model cannot be accurately known and hence it cannot be completely canceled. The proposed analysis, however, intuitively suggests that the arised internal forces will be lower in the case of a $G^{*}$ that satisfies condition 3) of Theorem 2

There exist cases, however, where it is required to achieve a desired non-zero internal force vector $h_{\text {int,d }} \in \mathbb{R}^{6 N}$ (e.g., to enforce contact maintenance). Clearly, $h_{\text {int,d }}$ must belong to the nullspace of $G$. In order to achieve $h_{\text {int }}=h_{\text {int,d }}$, one can add to (15) an appropriately defined extra term, as dictated in the following corollary.

Corollary 2: Let $h_{\text {int,d }} \in \operatorname{null}(G)$ be a desired internal force vector and $G^{*}=M G^{\top}\left(G M G^{\top}\right)^{-1}$, which satisfies (16). Then the control law $u^{\prime}=u+u_{\text {int,d, }}$, with

$$
u_{\mathrm{int}, \mathrm{d}}=\left(I_{6 N}-G^{*} G\right) h_{\mathrm{int}, \mathrm{d}},
$$

achieves points 1), 2) of Theorem 2 as well as $h_{\text {int }}=h_{\text {int,d }}$.

Proof: Since $h_{\mathrm{int}, \mathrm{d}} \in \operatorname{null}(G)$, the proof for points 1), $2)$ is identical with that of Theorem 2. Moreover, it holds that $h_{\text {int,d }} \in \operatorname{null}(G)=\operatorname{range}\left(A^{\top}\right)$ and hence $M^{-\frac{1}{2}} h_{\text {int,d }} \in$ $\operatorname{range}\left(M^{-\frac{1}{2}} A^{\top}\right)=\operatorname{range}\left(A M^{-\frac{1}{2}}\right)^{\dagger}$. Therefore, it holds that

$$
\begin{aligned}
\left(A M^{-\frac{1}{2}}\right)^{\dagger} A M^{-1} h_{\text {int,d }} & = \\
\left(A M^{-\frac{1}{2}}\right)^{\dagger} A M^{-\frac{1}{2}}\left(M^{-\frac{1}{2}} h_{\text {int, } \mathrm{d}}\right) & =M^{-\frac{1}{2}} h_{\text {int, } \mathrm{d} .}
\end{aligned}
$$

Substitution of 18 in (11) yields the resulting internal forces

$$
\begin{aligned}
h_{\text {int }} & =M^{\frac{1}{2}}\left(A M^{-\frac{1}{2}}\right)^{\dagger} A M^{-1}\left(I_{6 N}-M G^{\top}\left(G M G^{\top}\right)^{-1}\right) h_{\text {int,d }} \\
& =M^{\frac{1}{2}}\left(A M^{-\frac{1}{2}}\right)^{\dagger} A M^{-1} h_{\text {int, } \mathrm{d}}=M^{\frac{1}{2}} M^{-\frac{1}{2}} h_{\text {int, },}=h_{\text {int, },},
\end{aligned}
$$

where we have used (19) and the fact that $A G^{\top}=0$ from Theorem 1

Remark 3: Note that the expression (18) appears also in several works of the related literature for internal force regulation (e.g., [5], [6], [12], [14]). However, these works either do not provide any kind of formal guarantees that $h_{\text {int }}=h_{\text {int,d }}$ or do not give an explicit form for the choice of $G^{*}$. In fact, the main difference of our analysis with many of the previous works is the incorporation of the inertia matrix $M$ in the right inverse $G^{*}$, as dictated by the condition $(16)$.

\section{SIMULATION RESULTS}

We consider 4 UR 5 robots, rigidly grasping a rectangular object of dimensions $0.3 \times 0.3 \times 0.02 \mathrm{~m}^{3}$ and mass $0.5 \mathrm{~kg}$, in the realistic dynamic environment V-REP [23], as depicted in Fig. 2. We apply the algorithm (15) by using 3 different choices of $G^{*}$, namely, $G_{1}^{*}:=M G^{\top}\left(G M G^{\top}\right)^{-1}$, which satisfies the introduced condition (16), as well as the choices introduced in [12], [14] $G_{2}^{*}:=G^{\top}\left(G G^{\top}\right)^{-1}$, and $G_{3}^{*}:=W G^{\top}\left(G W G^{\top}\right)^{-1}$, with $W:=\operatorname{diag}\{\Delta, \ldots, \Delta\} \in$ $\mathbb{R}^{6 N \times 6 N}$, and $\Delta:=\left[\begin{array}{cc}0_{3 \times 3} & I_{3} \\ I_{3} & 0_{3 \times 3}\end{array}\right]$. The initial object condition is set to $x_{O}(0)=[-0.225,-0.6120,0.161,0,0,0]^{\top}$.

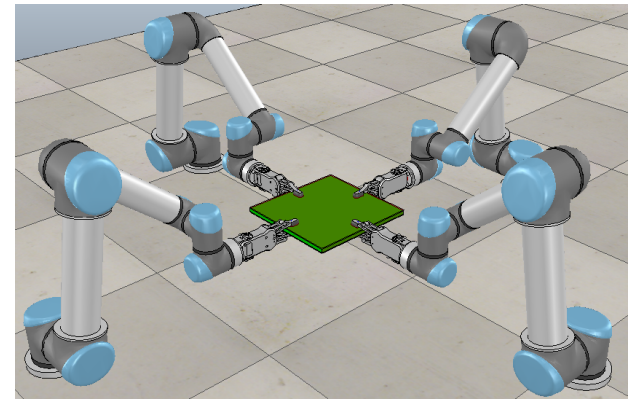

Fig. 2. Four UR5 robots rigidly grasping an object in V-REP environment.

The desired trajectory is chosen as $p_{\mathrm{d}}(t)=[-0.225+$ $0.1 \sin (t),-0.712+0.1 \cos (t), 0.16+0.1 \sin (t)]^{\top}, \eta_{\mathrm{d}}(t)=$ $[0.1 \sin (t), 0.1 \sin (0.5 t), 0.1 \sin (t)]^{\top} \mathrm{rad}$, from which we compute the respective rotation matrix $R_{\mathrm{d}}(t)$. The control gains are chosen as $K_{p}=60 I_{3}, k_{R}=0.06, K_{v}=K_{v_{1}}:=$ 7 diag $\{1,1,1,5,5,5\}$. The results for $t \in[0,20] \mathrm{sec}$ are depicted in Fig. 34 . Fig. 3 shows the evolution of the error norms $\left\|e_{p}(t)\right\|,\left\|e_{O}(t)\right\|$, and $\left\|e_{v}(t)\right\|$ for the three choices of $G^{*}$. As expected, the errors evolve close to zero for the three cases, implying thus asymptotic stability. Nevertheless, we observe significant oscillations of $e_{v}(t)$ in the cases of $G_{2}^{*}, G_{3}^{*}$, which can be attributed to the increased control input, as shown in Fig. 4 . More specifically, Fig. 4 depicts the agent input norm $\|\tau(t)\|$, where $\tau:=\left[\tau_{1}^{\top}, \tau_{2}^{\top}, \tau_{3}^{\top}, \tau_{4}^{\top}\right]^{\top}$, as well as the internal forces $h_{\text {int }}(t)$, computed using (11). It is evident that only $G_{1}^{*}$ achieves regulation of $h_{\text {int }}(t)$ close to zero, and also that $\|\tau(t)\|$ 's norm is minimum in the case of $G_{1}^{*}$, verifying the theoretical analysis.

Next, we increase slightly $K_{v}$ to $K_{v_{2}}$ := $7.25 \operatorname{diag}\{1,1,1,5,5,5\}$ and repeat the experiments, by depicting the results in Figs. 5,8. In particular, Fig. 5 shows the errors $\left\|e_{p}(t)\right\|,\left\|e_{o}(t)\right\|,\left\|e_{v}(t)\right\|$, and Fig. 6 shows $\|\tau(t)\|$ and $\left\|h_{\text {int }}(t)\right\|$ for $t \in[0,20]$ sec and the choice $G_{1}^{*}$, which still achieves asymptotic error stability as well as regulation of the internal forces close to zero. Fig. 7/8 provide the same information for the choices $G_{2}^{*}$ and $G_{3}^{*}$ and $t \in[0,7] \mathrm{sec}$, where an unstable behavior is observed. In particular, note that all the values for these cases exhibit oscillations of increasing magnitude. This further verifies the theoretical analysis, since $G_{2}^{*}$ and $G_{3}^{*}$ do not satisfy (16) and hence create undesired internal forces and increased control inputs that cause instability in a realistic setting (such as the V-Rep environment).

Finally, we derive a random force $h_{\text {int,d }}(t) \in$ $\operatorname{null}(G(x(t))), \forall t \geq 0$, and use the extra component (18) to achieve $h_{\text {int }}=h_{\text {int,d }}$, by using and comparing the choices $G_{1}^{*}$ and $G_{2}^{*}$, with $K_{v}=K_{v_{1}}$. Fig. 9 depicts the metric $\left\|e_{\text {int }}(t)\right\|, \forall t \in[0,20] \mathrm{sec}$, for $e_{\text {int }}:=h_{\text {int }}-h_{\text {int,d. }}$. As expected from the theoretical analysis, the choice $G_{1}^{*}$ has a much better performance, since it satisfies condition 16 . The non-perfect tracking can be attributed to the model uncertainties and hence the imperfect cancellation of the respective dynamic terms via (15), which is also why $h_{\text {int }}$, 

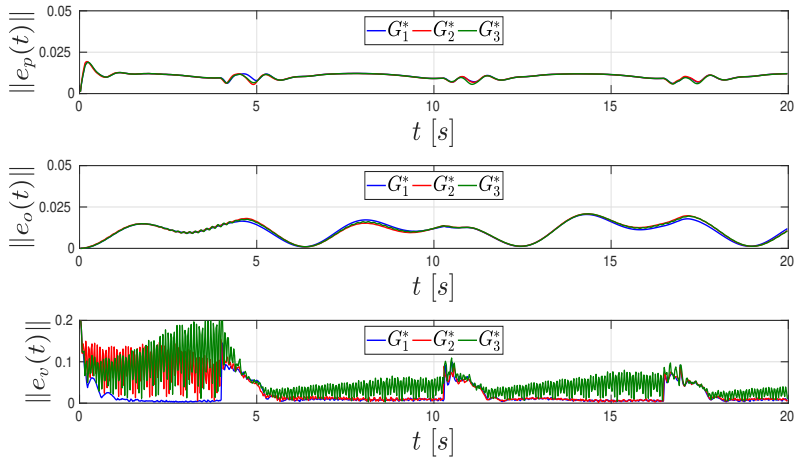

Fig. 3. The evolution of the error norms $\left\|e_{p}(t)\right\|,\left\|e_{O}(t)\right\|,\left\|e_{v}(t)\right\|$, $\forall t \in[0,20] \mathrm{sec}$ for the three choices of $G^{*}$ and $K_{v}=K_{v_{1}}$.
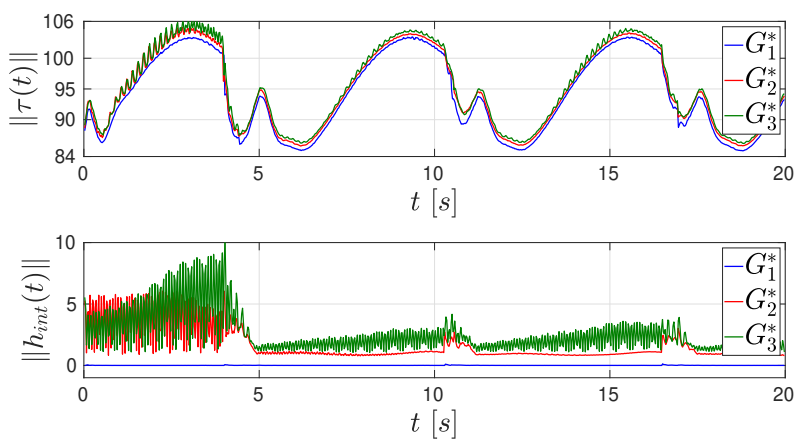

Fig. 4. The evolution of the norms $\|\tau(t)\|,\left\|h_{\text {int }}(t)\right\|, \forall t \in[0,20] \mathrm{sec}$ for the three choices of $G^{*}$ and $K_{v}=K_{v_{1}}$.

induced by $G_{1}^{*}$, are not identically zero in Figs. 4, 6 The accompanying video illustrates the aforementioned results.

\section{CONCLUSIONS}

This paper considers the energy-optimal rigid cooperative manipulation via minimization of the internal forces. By using a closed-form expression on the internal forces and a standard inverse-dynamics control law, we give novel conditions on the force distribution to the agents for internal forces avoidance. We also provide results on the provable achievement of nonzero internal forces. Simulation results verify the theoretical findings.
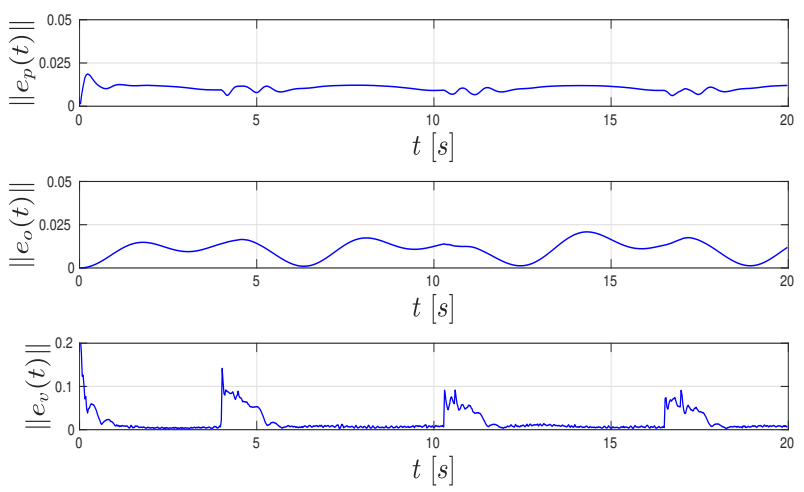

Fig. 5. The evolution of the error norms $\left\|e_{p}(t)\right\|,\left\|e_{O}(t)\right\|,\left\|e_{v}(t)\right\|$, $\forall t \in[0,20] \mathrm{sec}$ for $G^{*}=G_{1}^{*}$ and $K_{v}=K_{v_{2}}$.
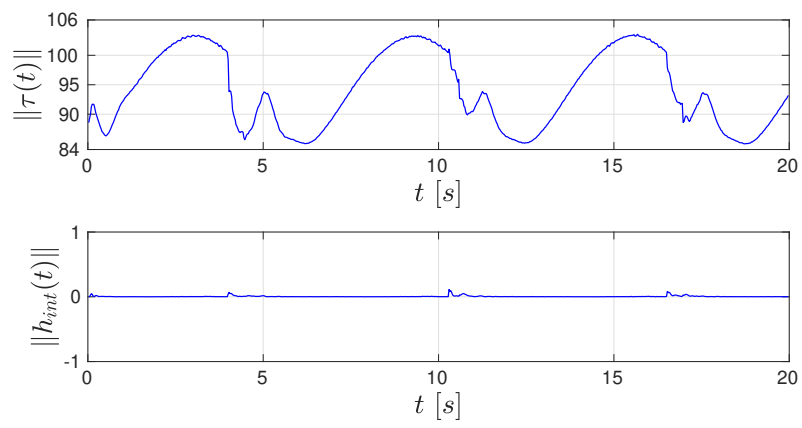

Fig. 6. The evolution of the norms $\|\tau(t)\|,\left\|h_{\text {int }}(t)\right\|, \forall t \in[0,20] \mathrm{sec}$ for $G^{*}=G_{1}^{*}$ and $K_{v}=K_{v_{2}}$.
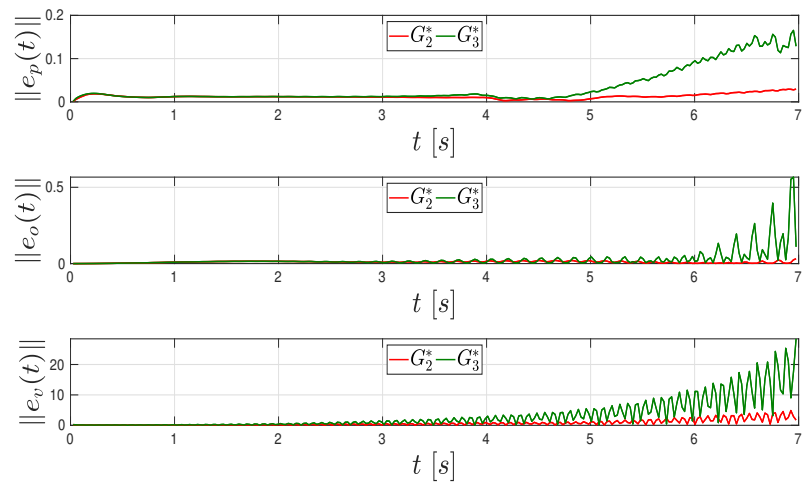

Fig. 7. The evolution of the error norms $\left\|e_{p}(t)\right\|,\left\|e_{O}(t)\right\|,\left\|e_{v}(t)\right\|$, $\forall t \in[0,7] \sec$ for $G_{2}^{*}, G_{3}^{*}$ and $K_{v}=K_{v_{2}}$.
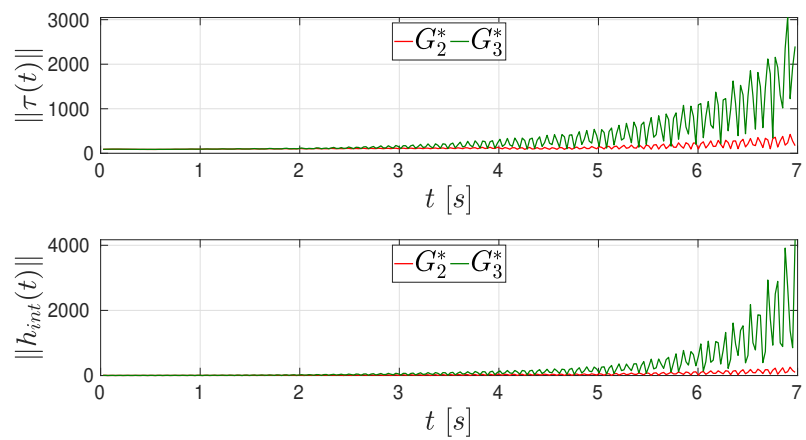

Fig. 8. The evolution of the norms $\|\tau(t)\|,\left\|h_{\text {int }}(t)\right\|, \forall t \in[0,7] \sec$ for $G_{2}^{*}, G_{3}^{*}$ and $K_{v}=K_{v_{2}}$.

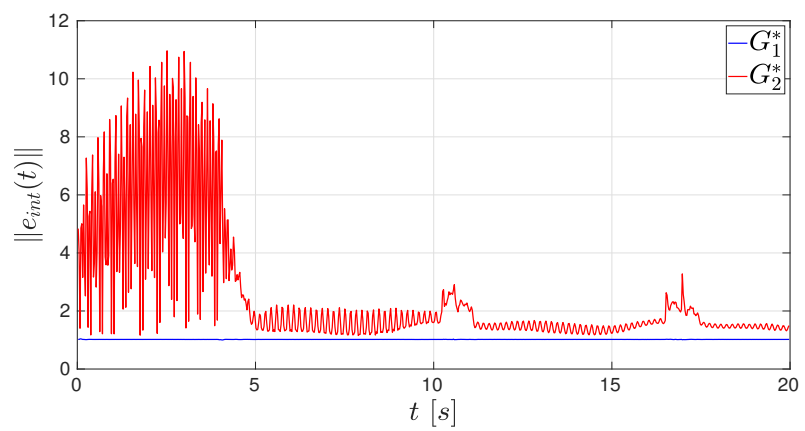

Fig. 9. The evolution of the norm $\left\|e_{\text {int }}(t)\right\|, \forall t \in[0,20] \sec$ for $G_{1}^{*}, G_{2}^{*}$ and $K_{v}=K_{v_{1}}$. 


\section{REFERENCES}

[1] O. Khatib, K. Yokoi, K. Chang, D. Ruspini, R. Holmberg, and A. Casal, "Decentralized cooperation between multiple manipulators," IEEE International Workshop on Robot and Human Communication, 1996.

[2] F. Caccavale, P. Chiacchio, and S. Chiaverini, "Task-space regulation of cooperative manipulators," Automatica, vol. 36, no. 6, 2000.

[3] F. Ficuciello, A. Romano, L. Villani, and B. Siciliano, "Cartesian impedance control of redundant manipulators for human-robot co-manipulation," IEEE/RSJ International Conference on Intelligent Robots and Systems (IROS), pp. 2120-2125, 2014.

[4] S. Erhart, D. Sieber, and S. Hirche, "An impedance-based control architecture for multi-robot cooperative dual-arm mobile manipulation," IEEE/RSJ International Conference on Intelligent Robots and Systems (IROS), pp. 315-322, 2013.

[5] A. Tsiamis, C. K. Verginis, C. P. Bechlioulis, and K. J. Kyriakopoulos, "Cooperative manipulation exploiting only implicit communication," IEEE/RSJ International Conference on Intelligent Robots and Systems (IROS), pp. 864-869, 2015.

[6] D. Heck, D. Kostić, A. Denasi, and H. Nijmeijer, "Internal and external force-based impedance control for cooperative manipulation," IEEE European Control Conference (ECC), pp. 2299-2304, 2013.

[7] W. Gueaieb, F. Karray, and S. Al-Sharhan, "A robust hybrid intelligent position/force control scheme for cooperative manipulators," Transactions on Mechatronics, vol. 12, no. 2, pp. 109-125, 2007.

[8] C. K. Verginis, M. Mastellaro, and D. V. Dimarogonas, "Robust cooperative manipulation without force/torque measurements: Control design and experiments," IEEE Transactions on Control Systems Technology, pp. 1-17, 2019.

[9] A.-N. Ponce-Hinestroza, J.-A. Castro-Castro, H.-I. Guerrero-Reyes, V. Parra-Vega, and E. Olguỳn-Dỳaz, "Cooperative redundant omnidirectional mobile manipulators: Model-free decentralized integral sliding modes and passive velocity fields," IEEE International Conference on Robotics and Automation (ICRA), pp. 2375-2380, 2016.

[10] A. Marino, "Distributed adaptive control of networked cooperative mobile manipulators," IEEE Transactions on Control Systems Technology, vol. 26, no. 5, pp. 1646-1660, 2017.
[11] J. Gregory, A. Olivares, and E. Staffetti, "Energy-optimal trajectory planning for robot manipulators with holonomic constraints," Systems \& Control Letters, vol. 61, no. 2, pp. 279-291, 2012

[12] I. D. Walker, R. A. Freeman, and S. I. Marcus, "Analysis of motion and internal loading of objects grasped by multiple cooperating manipulators," The International journal of robotics research, vol. 10 no. 4, pp. 396-409, 1991.

[13] D. Williams and O. Khatib, "The virtual linkage: a model for internal forces in multi-grasp manipulation," IEEE International Conference on Robotics and Automation, vol. 1, pp. 1025-1030, 1993.

[14] J. H. Chung, B.-Y. Y. W. K., and Kim, "Analysis of internal loading at multiple robotic systems," Journal of mechanical science and technology, vol. 19, no. 8, pp. 1554-1567, 2005.

[15] S. Erhart and S. Hirche, "Internal force analysis and load distribution for cooperative multi-robot manipulation," IEEE Transactions on Robotics, vol. 31, no. 5, pp. 1238-1243, 2015.

[16] — "Model and analysis of the interaction dynamics in cooperative manipulation tasks," Transactions on Robotics, vol. 32, no. 3, 2016.

[17] B. Siciliano, L. Sciavicco, L. Villani, and G. Oriolo, Robotics: modelling, planning and control. Springer Science \& Business Media, 2010.

[18] R. Kalaba and F. Udwadia, "Equations of motion for nonholonomic, constrained dynamical systems via gausss principle," Journal of Applied Mechanics, vol. 60, no. 3, pp. 662-668, 1993.

[19] A. Albert, Regression and the Moore-Penrose Pseudoinverse. Elsevier, 1972.

[20] C. K. Verginis, A. Nikou, and D. V. Dimarogonas, "Robust formation control in se(3) for tree-graph structures with prescribed transient and steady state performance," Automatica, vol. 103, pp. 538-548, 2018.

[21] H. K. Khalil, "Noninear systems," Prentice-Hall, New Jersey, vol. 2 no. 5 , pp. 5-1, 1996

[22] C. K. Verginis, D. Zelazo, and D. V. Dimarogonas, "Cooperative manipulation via internal force regulation: A rigidity theory perspective,' Under Review. Arxiv Link: https://arxiv.org/pdf/1911.01297.pdf, 2019.

[23] E. Rohmer, S. P. Singh, and M. Freese, "V-rep: a versatile and scalable robot simulation framework," IEEE/RSJ International Conference on Intelligent Robots and Systems (IROS), 2013. 